\title{
Use of an implantable cardioverter defibrillator in a patient with a rate responsive pacemaker
}

\author{
R Chamberlain-Webber, I Rankin, R Sutton
}

\begin{abstract}
A patient dependent on a pacemaker and with a rate responsive unit presented with symptomatic ventricular tachycardia despite drug treatment. He was managed with a third generation implantable cardioverter defibrillator, while rate responsive pacing was maintained.
\end{abstract}

(Br Heart F 1994;71:191-192)

Reports of interactions between implanted devices in cardiology are increasing as technology progresses. We present a case in which a patient with a rate responsive pacemaker underwent implantation of a cardioverter defibrillator for ventricular tachycardia.

\section{Case history}

A retired 60 year old man was referred with recent onset symptomatic ventricular tachycardia, angina, and presyncope. He had a history of ischaemic heart disease and after a myocardial infarction in 1979 and coronary bypass surgery four years later, had a VVIR pacemaker implanted in 1985 for atrioventricular block. He remained well in follow up until 1990 when he underwent an elective change of pacemaker because of depletion of the battery. After his original implantation he had become dependent on the pacemaker. The importance of rate response to the patient was apparent when his pacemaker reverted to end of life mode without rate response and produced dyspnoea limiting the patient's active lifestyle. The patient had not experienced angina since his bypass operation as he was being treated with $\beta$ blockers and oral nitrates.

On presentation at the referring hospital, initial treatment with lignocaine and amiodarone was unsuccessful and the patient needed cardioversion. He remained on oral amiodarone after a 1.2 gm intravenous loading dose and was transferred for further investigation. Amiodarone concentrations were not measured. Electrophysiological study one week after presentation showed an easily inducible symptomatic sustained monomorphic ventricular tachycardia, identical to the clinical tachycardia although slower. Overdrive pacing ended the tachycar- dia without acceleration. Left ventricular angiography showed a dilated, hypokinetic left ventricle with -native three vessel disease. The ejection fraction was $40 \%$ and all bypass grafts were blocked. Further bypass grafting was not considered feasible and in view of his ischaemic aetiology further drug trials were thought inappropriate.

Because of the ease with which ventricular tachycardia could be induced and persistence of symptoms despite amiodarone, the patient was fitted with an endocardial cardioverter defibrillator (Medtronic PCD model 7217B), and rate responsive pacing was retained (figure). His existing pacemaker was replaced with a bipolar VVIR system with an active fixation lead. This system was tested during the operation with an external tachycardia control device for detection and delivery of stimuli before final implantation. Telemetry was used to check for oversensing when the pacemaker was programmed to basic rates of 60 and 120 beats $/ \mathrm{min}$, to simulate a high activity rate. Ventricular tachycardia was then induced so that appropriate inhibition of the pacemaker and reliable ending of tachycardia with antitachycardia pacing could be assessed. This was repeated satisfactorily while the pacemaker was set at 120

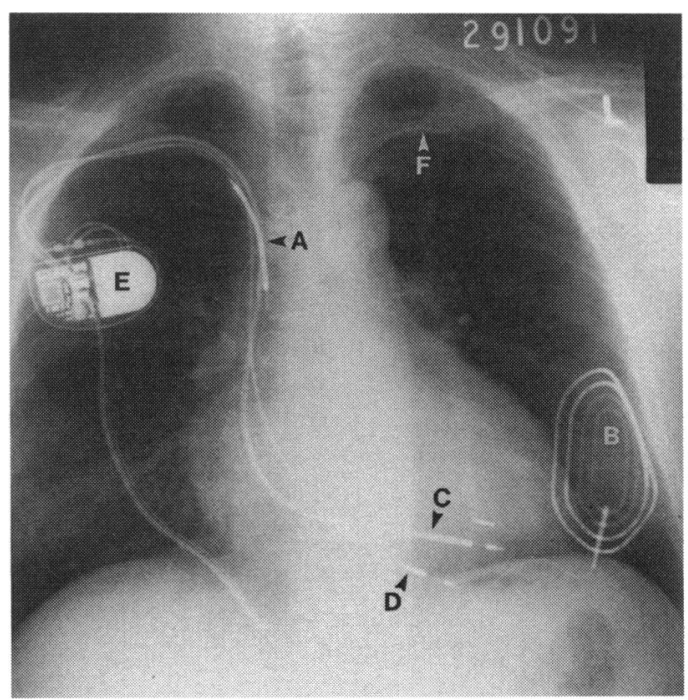

Figure 1 Chest $x$ ray film after implantation, showing superior vena caval $(A)$, subcutaneous patch $(B)$, and right ventricular $(C)$ defibrillator electrodes, with active fixation pacing wire (D) from the pacemaker(E) in the right pectoral region. The cut ventricular lead from the previous
left sided pacemaker $(F)$ is also visible. The cardioverter defibrillator is not seen on this film. 
beats/min, simulating a high level of activity. Tests were completed by delivery of maximal shock during tachycardia to ensure that the function of the pacemaker was maintained. Ventricular fibrillation could not be induced despite aggressive stimulation. After final implantation, progress was uneventful.

Before discharge a repeat of the electrophysiological study confirmed satisfactory function of the device with respect to antitachycardia pacing, cardioversion, and VVI pacing over a range of rates $(60-120$ beats/min). The patient then underwent exercise treadmill tests to assess function of the cardioverter defibrillator with the Activitrax rate response programmed $\mathrm{ON}$. This was satisfactory with the patient achieving the maximal pacing rate (which was set less than the rate of ventricular tachycardia). This was discontinued due to fatigue. The electrocardiogram and the telemetered marker channel of the cardioverter defibrillator were monitored continuously during the test. At follow up at six months the patient remains well with no clinical recurrence of his ventricular tachycardia and no detected problems on investigation of the device. He has returned to his former, active lifestyle, including swimming and golf.

\section{Discussion}

With increasing use worldwide of implantable cardioverter defibrillators, it is becoming more likely that recipients will already have or need some form of pacemaker for conduction tissue or sinus node disease. ${ }^{1}$ Current designs of cardioverter defibrillators incorporate the ability to provide antitachycardia pacing and many also have back up for ventricular pacing. At present no units are available that provide rate responsive or dual chamber pacing although these developments may not be far away.

Earlier published reports described the scope of potential and actual interactions between implanted cardioverter defibrillators and pacemakers. ${ }^{2-5}$ These include difficulties in counting the pacing spikes twice by the cardioverter defibrillator circuits ${ }^{6}$ and problems with inhibition of the cardioverter defibrillator by large unipolar pacing spikes. ${ }^{3}$

This case report documents the use of a rate responsive pacemaker with a third generation cardioverter defibrillator. Although use of a rate responsive pacemaker with a cardioverter defibrillator has been previously described, ${ }^{7}$ this was with a first generation epicardial system without programmable antitachycardia pacing, and there is no description of exercise testing after implantation to assess interaction between devices.

Previously reported difficulties with combined implantations were dealt with in several ways, including use of a bipolar pacing system, positioning of the pacing electrode to minimise the pacing artefact sensed by the cardioverter defibrillator, and adjustment of the sensitivity of the cardioverter defibrillator so that inhibition of output during ventricular tachycardia did not occur, even with inappropriate pacing.

Novel aspects of this case, by contrast with previous case reports that deal almost exclusively with epicardial systems of earlier design, are the use of combined systems in a pacemaker dependent patient, maintenance of rate response, and the use of a third generation transvenous cardioverter defibrillator.

Points of note arising from our experience, as well as those previously published are:

(a) The ability to use endocardial pacing systems with transvenous cardioverter defibrillators.

(b) The need for adequate assessment after implantation including exercise testing where a rate responsive pacemaker is implanted, and to set a suitable upper rate for pacing that will not trigger detection of tachycardia.

(c) The need for close collaboration between the clinical team and the manufacturer's technical support group at an early stage to reduce the possibility of adverse interactions.

1 Ruffy R, Lal R, Kouchoukos NT, Kim SS. Combined bipolar dual chamber pacing and automatic implantable cardioverter/defibrillator. $\mathcal{f} \mathrm{Am}$ Coll Cardiol 1986;7: 933-7.

2 Singer I, Guarnieri $T$, Kupersmith J. Implanted automatic defibrillators: effects of drugs and pacemakers. PACE defibrillators: effects

3 Kim SG, Furman S, Waspe LE, Brodman R, Fisher JD. Unipolar pacer artifacts induced failure of an automatic implantable cardioverter/defibrillator to detect ventricular fibrillation. Am f Cardiol 1986;57:880-1.

4 Slepian M, Levine JH, Watkins Jr L, Brinker J, Guarnieri T. Automatic cardioverter defibrillator/permanent pacemaker interaction: loss of pacemaker capture following AICD discharge. PACE 1987;10:1194-7.

5 Barold SS, Falkoff MD, Ong LS, Heinle RA. Interference in cardiac pacemakers: exogenous sources. In: El-Sherif N, Samet P, eds. Cardiac pacing and electrophysiology. Philadelphia: Saunders WB, 1991;608-33.

6 Cohen AI, Wish MH, Fletcher RD, et al. The use and interaction of permanent pacemakers and the automatic implantable cardioverter defibrillator. $P A C E$ 1988;11: 704-11.

7 Epstein AE, Kay N, Plumb VJ, Shepard RB, Kirklin KJ. Combined automatic cardioverter-defibrillator and pacemaker systems: implantation techniques and followup. $¥ \mathrm{Am}$ Coll Cardiol 1989;13:121-31. 